\title{
Differentiating ERAN and MMN: An ERP study
}

\author{
Stefan Koelsch, I,CA Thomas C. Gunter,' Erich Schröger, ${ }^{2}$ Mari Tervaniemi,, ${ }^{3}$ Daniela Sammler ${ }^{1,2}$ and \\ Angela D. Friederici ${ }^{1}$
}

'Max Planck Institute of Cognitive Neuroscience, Stephanstr. Ia, D-04I03 Leipzig; ${ }^{2}$ Institute of General Psychology, Leipzig, Germany; ${ }^{3}$ Cognitive Brain Research Unit, Helsinki, Finland

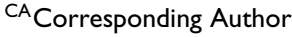

Received I4 February 200I; accepted 27 February 200 I

\begin{abstract}
In the present study, the early right-anterior negativity (ERAN) elicited by harmonically inappropriate chords during listening to music was compared to the frequency mismatch negativity (MMN) and the abstract-feature MMN. Results revealed that the amplitude of the ERAN, in contrast to the MMN, is specifically dependent on the degree of harmonic appropriateness. Thus, the ERAN is correlated with the cognitive processing of complex rule-based information, i.e. with the application of music-syntactic rules. Moreover, results showed that the ERAN, compared to the abstract-feature MMN, had
\end{abstract}

Key words: Auditory processing; EEG; ERAN; ERP; MMN; Music both a longer latency, and a larger amplitude. The combined findings indicate that ERAN and MMN reflect different mechanisms of pre-attentive irregularity detection, and that, although both components have several features in common, the ERAN does not easily fit into the classical MMN framework. The present ERPs thus provide evidence for a differentiation of cognitive processes underlying the fast and pre-attentive processing of auditory information. NeuroReport 12:1385-1389 (C) 200I Lippincott Williams \& Wilkins.

\section{INTRODUCTION}

Accurate pitch perception is a prerequisite for the processing of melodic, harmonic, and prosodic aspects of both language and music. Recently, neural dynamics underlying pitch processing within a musical context have been extensively investigated by recording the mismatch negativity (MMN) $[1,2]$, the right anterior-temporal negativity (RATN) [3], and the early right-anterior negativity (ERAN) [4-6], which are components of the auditory event-related potential (ERP). The present study aimed at differentiating MMN and ERAN.

In previous studies investigating the perception of major-minor (i.e. Western) tonal music, harmonically inappropriate chords presented within a musical chord sequence elicited a negativity in the ERP which was maximal around $200 \mathrm{~ms}$ and right-anteriorly predominant $[4,5]$. Due to its early latency with respect to the RATN, and due to its similarity to the early left anterior negativity (ELAN) [7], this ERP component was termed the early right-anterior negativity, or ERAN. With respect to their functional significance, the RATN and the ERAN are suggested to reflect the processing of musical syntax, whereas the ELAN is known to be elicited by word category violations and therefore taken to reflect syntactic language processing.

In the studies of Koelsch et al. [4-6], harmonically inappropriate chords did not represent a physical deviancy with respect to the preceding chords. Thus, the ERAN is not a frequency MMN which is known to be elicited only when a deviant tone, or chord, is preceded by a few standard tones or chords with identical frequency $[8,9]$. Notably, both harmonically appropriate and inappropriate chords were consonant, major chords. It was only the surrounding musical context that made some chords with respect to principles and rules described by the theory of harmony inappropriate (see below).

Nevertheless, the ERAN is reminiscent of the MMN: (a) both ERAN and MMN have a similar time-course and scalp-distribution, (b) the amplitudes of both ERAN and MMN increase with the amount of violation and are connected to behavioral discrimination performance, and (c) both MMN and ERAN can be elicited pre-attentively $[5,8,9]$. Moreover, in previous experiments the ERAN was elicited in a paradigm which has similarities to the auditory oddball paradigm, a paradigm often used to elicit an MMN.

In the present study, chord sequences were presented to the participants, each sequence consisting of five chords, and one sequence directly following the other (middle of Fig. 1). Most of the sequences consisted of in-key chords only, but infrequently chords at the third or at the fifth position of the sequences were Neapolitan sixth chords. Neapolitan chords (in C major: $\mathrm{f}-\mathrm{a}$ flat-d flat; Fig. 1) contain out-of-key notes (in C major: a flat and d flat) with respect to the harmonic context established by the preceding in-key chords. Following the theory of harmony, 

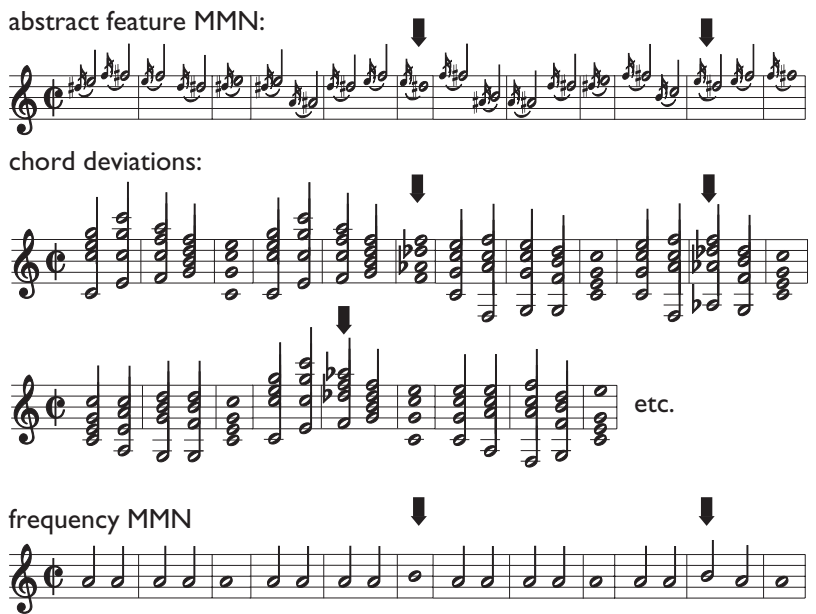

Fig. I. Examples of stimuli. In all blocks, stimuli were presented with the same time-course, loudness, and probability of deviant events (deviant events are indicated by the arrows).

Neapolitans may be considered as a variation of the subdominant (a minor subdominant with a minor sixth instead of a fifth). Neapolitans at the fifth position are perceived as more inappropriate than when they are presented at the third position for two reasons: (a) because of the musical context buildup, the tonal expectancies of listeners are more specific at the end of a sequence $[10,11]$, and (b) since Neapolitans function in music- theory as subdominant variation, a Neapolitan at the third position is fairly suitable (since a subdominant is appropriate), whereas a Neapolitan at the fifth position (where a tonic chord is appropriate only) is inappropriate; it has been shown that harmonically appropriate chord functions are expected to a higher degree compared to inappropriate chords [10,11].

Notably, harmonic expectancies of listeners follow principles which correspond to the harmonic relations of different chords (and keys, respectively). These relationships form the basis of the major-minor tonal system and are described by the theory of harmony. The principles which govern harmonic expectancies have been described in detail as a hierarchy of harmonic stability [11]. In previous music experiments employing an experimental paradigm similar to that of the present study [4-6] it could be shown that the degree of harmonic incongruity (i.e. the degree of harmonic expectancy violation) is reflected in the amplitude of the ERAN: The ERAN is smaller when elicited by Neapolitans at the third position of a chord sequence compared to the fifth position. That is, the elicitation and amplitude modulation of the ERAN can be explained on the basis of music theory, or, in other words, the ERAN may reflect cognitive processes which refer to a complex rule system (which may be taken as musical syntax $[4,6,10-12])$. This would contrast the MMN, which is known to be elicited either by physical deviance, or by rather simple abstract feature deviances which do not refer to a system of complex rules.

However, although the ERAN cannot be a frequency MMN (see above), the ERAN could in principle be an MMN elicited by the abstract feature 'harmonically appro- priate/inappropriate' [13-16]. If so, the amplitude modulation of the ERAN (third versus fifth position of a chord sequence) would not be due to any complex rule-based processing of harmonic information, but merely be dependent on the position within a stimulus train (i.e. within a sequence of acoustic events), regardless of the buildup of a musical context.

In order to test whether the ERAN is an abstract feature MMN, participants of the present study were presented with acoustic events containing an abstract feature (tone pairs raising (standard) or falling (deviant) in pitch, see Fig. 1), but not building up a context towards the end of each stimulus sequence. If the processes reflected in the ERAN are the same as those reflected in the MMN, the amplitude of the abstract feature MMN should, like the amplitude of the ERAN, increase towards the end of a stimulus sequence. Three blocks were conducted: an abstract feature MMN block, a block with chord sequences, and a frequency MMN block (with single tones, Fig. 1). In all three blocks, stimuli were presented with the same time-course and the same probability of deviant events.

\section{MATERIALS AND METHODS}

Subjects: Twenty-eight right-handed and normal-hearing subjects (aged 19-28 years, mean 23.4, 15 of them females) participated in the experiment. Subjects were non-musicians, i.e. they had never learned to play an instrument or professional singing, and they did not have any special musical education besides normal school education.

Stimuli: All stimuli were played under computerized control via MIDI with a piano sound on a Roland JV-2080 synthesizer. In all blocks, each stimulus sequence consisted of five events, presentation time (PT) of events 1-4 was $600 \mathrm{~ms}$, of the fifth event $1200 \mathrm{~ms}$ (in the first block, the first tone of each tone-pair had a PT of $100 \mathrm{~ms}$, i.e. PT of the second tone was $500 \mathrm{~ms}$ ( $1100 \mathrm{~ms}$ at the fifth position) respectively). Deviant events (in the first block falling tonepairs, in the second block Neapolitan chords, in the third block tones with different frequency) occurred in some sequences randomly at either the third $(p=0.2)$ or fifth position $(p=0.2)$. All chords and tones had the same loundness and the same decay of loudness, there was no silent period between events or sequences; one sequence directly followed the other (Fig. 1). In each block, the same deviant events were employed at the third and fifth position, i.e. deviant events were in each block on average physically identical. Sequences containing a deviant event were always preceded by a sequence exclusively consisting of standards. Chords and tones were played with $\sim 55 \mathrm{~dB}$ SPL. In each block, 255 sequences were presented, resulting in a block duration of $15 \mathrm{~min}$.

Task: In all blocks, subjects were playing a video-game under the instruction to ignore all acoustic stimuli.

In Block 1 (abstract feature MMN), standard stimuli were single-tone pairs raising in pitch, deviant pairs were falling in pitch (top of Fig. 1). The pitch difference of two tones of a pair was one semitone ( $\sim 6 \%$ frequency difference). Three different tone pairs could occur at the first position of a sequence, six at the second, six standards at the third, three deviants at the third, three at the fourth, 
two standards at the fifth, and three deviants at the fifth position (3/6/6(3)/3/2(3)). The MMN was measured from the onset of the second tone of a pair.

In block 2 (ERAN), all sequences consisted of five chords that began with a tonic-chord. Chords at the second position were tonic, subdominant, mediant, or submediant; at the third position: subdominant, Neapolitan chord, dominant, or dominant six-four chord; at the fourth position: dominant seventh chord; at the fifth position: tonic or Neapolitan chord [17]. All chords were presented in different chordings (e.g. with the root, the third, the fifth, and the seventh in the top voice), leading to a pool of 108 different chord sequences. Importantly, the number of physically different chords possible at each position of a sequence equalled the number of different tone pairs of Block 1 (3/6/6(deviant: 3 different Neapolitans)/3/2(deviant: 3 different Neapolitans)). Part-writing was according to the classical rules of harmony [17].

In block 3 (frequency MMN), Standards were single tones with a frequency of $440 \mathrm{~Hz}$, deviant tones had a frequency of $496 \mathrm{~Hz}$.

Data analysis: The EEG was recorded with nose-reference from 41 electrodes of the extended 10-20 system (sampling rate $250 \mathrm{~Hz}$ ). All EEG data were filtered off-line with a bandpass filter $(0.25-25 \mathrm{~Hz}, 1001$ points, FIR). Artifacts caused by drifts or body movements were eliminated by rejecting EEG data of all blocks whenever the standard deviation within any $600 \mathrm{~ms}$ or $200 \mathrm{~ms}$ interval of all data $>25 \mu \mathrm{V}$ at any electrode. Eye artifacts were rejected whenever the s.d. within any $200 \mathrm{~ms}$ interval of all data exceeded $30 \mu \mathrm{V}$ at either the vertical or the horizontal EOG. Baseline of ERPs was -50 to $0 \mathrm{~ms}$ relative to stimulus onset. ERPs were analyzed by repeated measures ANOVAs as univariate tests of hypotheses for within subjects effects. Two anterior regions of interest (ROIs) were computed: left (mean of F3, FC3, F5, FC5, AF3, AF7) and right (mean of F4, FC4, F6, FC6, AF4, AF8). If not separately indicated, ANOVAs were conducted with factors condition (standard $\times$ deviant), position within the sequences (third $\times$ fifth position), and hemisphere (left $\times$ right ROIs).

\section{RESULTS}

In the abstract feature MMN block, deviant tone pairs presented at both the third and the fifth position elicited an abstract feature MMN (Fig. 2, left). The latency of the MMN was at both the third and the fifth position around $160 \mathrm{~ms}$ (measured from the onset of the second tone of the abstract feature MMN
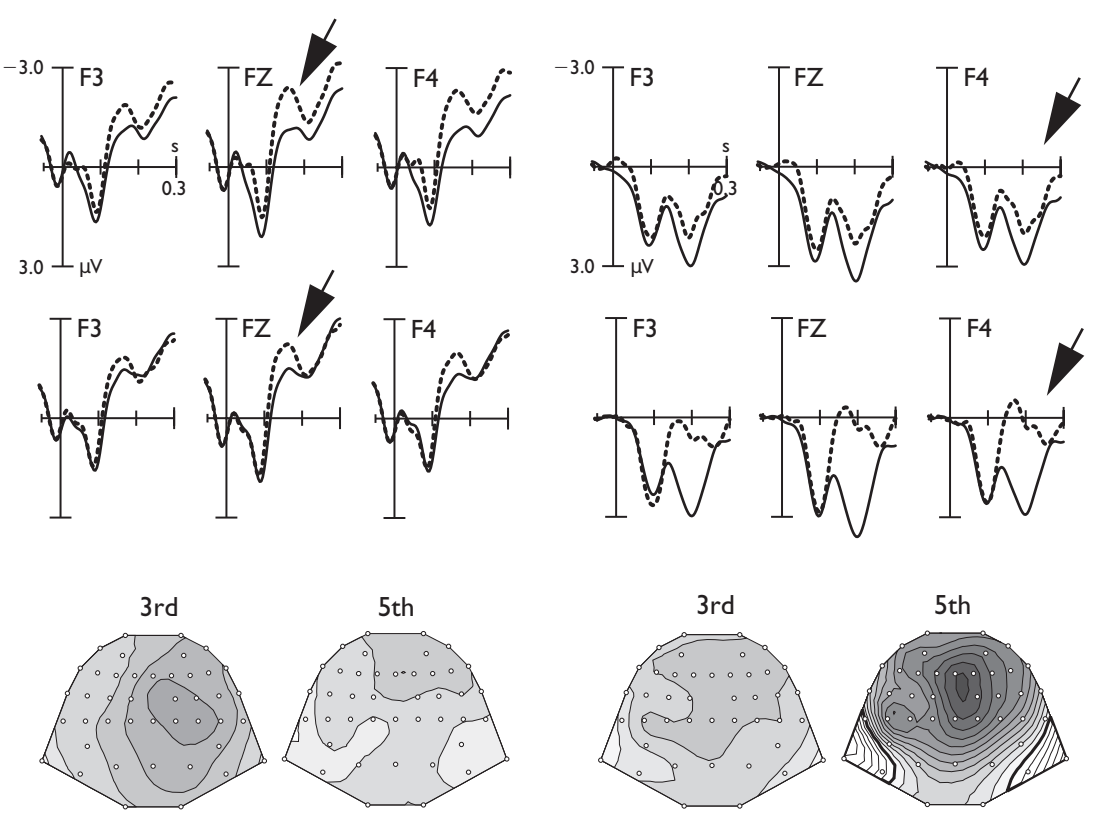
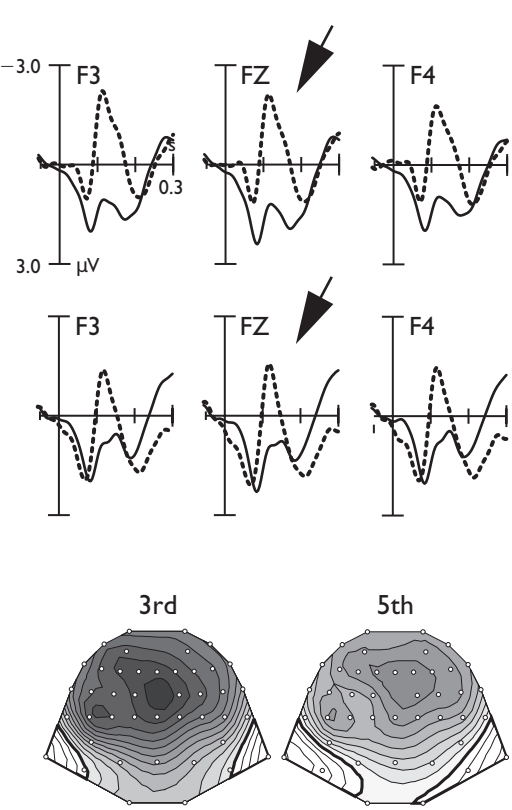

frequency MMN
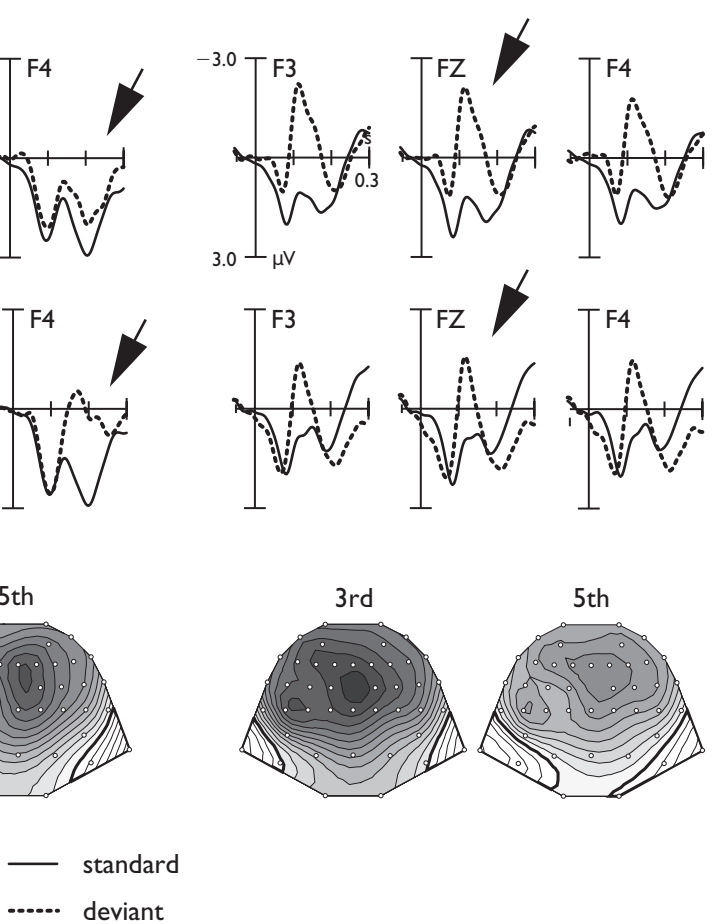

Fig. 2. ERPs elicited at frontal electrode sites by stimuli at the third (top row) and fifth (second row) position, separately for the abstract feature MMN block, the block with chord sequences, and the frequency MMN block. Vertical line indicates the onset of the deviant stimulus (in the abstract feature MMN block: second tone of a tone pair). Bottom row: Potential maps of abstract feature MMN (standard subtracted from deviant), ERAN (harmonic appropriate chords subtracted from Neapolitan chords), and frequency MMN (standard subtracted from deviant), separately for third and fifth position. Maps were calculated using the data from all $4 \mathrm{I}$ electrodes and interpolated over time windows from I25 to I85 ms (abstract feature MMN), 170-230 ms (ERAN) and 90-150 ms (frequency MMN), polarity inversions are bordered by thick lines. In contrast to the ERAN, the MMNs did not differ in amplitude between position 3 and 5, indicating that the ERAN reflects context-dependent musical processing. Moreover, the ERAN elicited at the fifth position had a later latency, but larger amplitude than the abstract feature MMN. 
tone pair), the amplitudes of the MMN did not differ between both positions (see also Fig. 3); no polarity inversion was visible at mastoidal sites. An ANOVA conducted for a time window from 125 to $185 \mathrm{~ms}$ revealed an effect of condition $(\mathrm{F}(1,26)=15.54, p<0.0005)$, an interaction between factors condition and hemisphere $(\mathrm{F}(1,26)=9.12$, $p<0.006)$, and no interaction between factors condition and position.

In the second block (chord-sequences), Neapolitan chords at both the third and the fifth position elicited an ERAN with a latency around $200 \mathrm{~ms}$, the ERAN at the fifth position showed a clear polarity inversion at mastoidal sites (Fig. 2, middle, the polarity inversion is indicated in the potential map). The ERAN was distinctly larger at the fifth than at the third position (see also Fig. 3). The amplitude of the ERAN elicited at the fifth position was clearly larger than the amplitude of the abstract feature MMN elicited at the fifth position in the first block. An ANOVA for the data of the second block (time window $170-230 \mathrm{~ms})$ revealed an effect of condition $(\mathrm{F}(1,26)=$ 38.53, $p<0.0001)$, an interaction between factors condition and hemisphere $(\mathrm{F}(1,26)=10.24, p<0.005)$, and an interaction between factors condition and position $(F(1,26)=$ 9.32, $p<0.006)$. ANOVAs with factor condition conducted separately for the third and the fifth position revealed an effect of condition at both third and fifth position (third position: $\mathrm{F}(1,26)=4.74, p<0.05$; fifth position: $\mathrm{F}(1,26)=$ $36.33, p<0.0001)$. An ANOVA of the data from the fifth position from blocks 1 and 2 with factors condition and block $(1 \times 2)$, testing the amplitude difference between the abstract feature MMN (125-185 ms) and the ERAN (170$230 \mathrm{~ms})$, revealed an interaction between the two factors $(\mathrm{F}(1,26)=12.43, p<0.002)$.

In the frequency MMN block, deviant tones elicited a frequency $\mathrm{MMN}$ at both the third and the fifth position, with a latency of around $100 \mathrm{~ms}$, with clear polarity inversion at mastoidal sites, and with virtually the same amplitude at the third and fifth position (Fig. 2, right, and Fig. 3). An ANOVA for the time window from 90 to $150 \mathrm{~ms}$ revealed an effect of condition $(\mathrm{F}(1,26)=62.27, p<0.0001)$, with no interaction between factors condition and position (no interaction was yielded between factors condition and hemisphere, although a slight right hemispheric preponderance is visible in the potential maps).

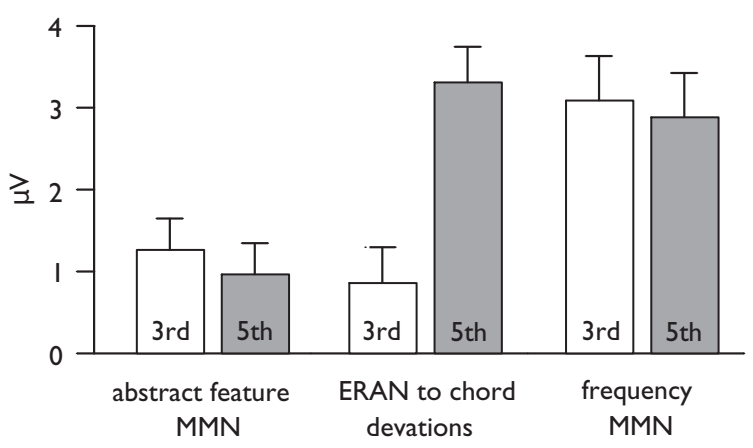

Fig. 3. Amplitudes of abstract-feature MMN (left), ERAN (middle) and MMN (right), separately for positions 3 (white bars) and 5 (gray bars). Latency of the abstract feature MMN was $160 \mathrm{~ms}$, of the ERAN $200 \mathrm{~ms}$, and of the frequency MMN $100 \mathrm{~ms}$.

\section{DISCUSSION}

The present study investigated the pre-attentively activated neural mechanisms of an auditory deviance detection by comparing the ERP response to an abstract sound change, a change of harmonic appropriateness of chords, and a pitch change. Only the ERAN (elicited by the harmonically incongruous chords in the second block) differed significantly in amplitude between third and fifth position of a sequence. Neither abstract feature MMN nor frequency MMN (elicited in a non-musical context) showed an amplitude modulation between these positions. Since only the in-key chords of the second block built up a musical context towards the end of each sequence, this finding indicates that the amplitude of the ERAN is specifically correlated with the degree of harmonic incongruity induced by a preceding musical context. The present results thus demonstrate that the ERAN reflects processing of auditory information that refers to a complex rule-based system, namely rules inherent in the major-minor tonal system which are far more complex than those known to elicit a physical or an abstract feature MMN.

Notably, the ERAN elicited at the fifth position had a longer latency, and a larger amplitude than the abstract feature MMN. The longer latency could easily be explained within the classical MMN framework if one assumes that the harmonic incongruities are more complex, and thus more difficult to differentiate than the deviant tone-pairs: The MMN is known to have a longer latency when stimuli are more difficult to differentiate $[8,18]$. Then, however, the ERAN should also be smaller in amplitude than the MMN [18]. This was not the case: on the contrary, the ERAN was distinctly larger than the abstract feature MMN, indicating that the ERAN does not reflect the same cognitive processes that underlie the MMN. The present ERPs thus provide evidence for a differentiation of cognitive processes underlying a pre-attentive processing of auditory information in (a) sensory memory processes on the one side, and (b) relatively higher cognitive processing of complex rule-based information on the other. The fact that the ERAN specifically correlates with the processing of complex rules of major-minor tonal music justifies the particular term ERAN for the effects observed.

It is important to note that MMN, ERAN, and ELAN all belong to a familiy of peri-sylvian (see also below) negativities which reflect the processing of irregularities of auditory input. The present study supports the notion that there are, however, considerable differences with respect to the cognitive processes and the neuronal structures mediating the processing of a physical irregularity like frequency on the one hand, and a language or music syntactic violation on the other. The fact that an MMN can also be elicited by abstract features might indicate that all components (physical MMNs, abstract feature MMN, ERAN and ELAN) reflect stages on a continuum from rather simple (physical) to fairly complex (syntactic) auditory feature processing; this consideration might suggest an expansion of the classical MMN framework. Notably, this consideration is supported by functional neuroanatomical findings, which indicate that the more simple features seem primarily to be generated in (or in the close vicinity of) primary auditory cortical areas, with relatively small contributions from the frontal areas [19-22]. In contrast, the processing 
of features which refer to a complex rule system (as reflected in ERAN and ELAN) appears to involve more frontal, and less primary auditory structures [6,23] (a recent study from Koelsch et al. [6] revealed that the ERAN is mainly generated in the inferior part of BA 44 bilaterally).

Finally, the ERAN was (as the MMNs) elicited under a condition in which participants were instructed to ignore the chords, supporting the hypothesis that the ERAN reflects, like the MMN [8], pre-attentive neural processes [5]. Taken together, the present results thus support the hypothesis of a high adaptability and flexibility of preattentive processes in the human brain [4].

\section{CONCLUSION}

The present results demonstrate that the ERAN reflects cognitive operations connected to the processing of complex rule-based musical information, in contrast to the MMN, which is known to reflect mainly sensory memory operations. This finding is important for several reasons. First, it indicates that partly different neuronal processes underlie the generation of ERAN and MMN (although both components share several features). Since both MMN and ERAN can be elicited pre-attentively, the present data provide evidence for a differentiation of fast and preattentive neural mechanisms underlying auditory deviance detection in the human brain. Second, although the ERAN does not easily fit into the classical MMN framework, both components fit into one concept if one considers that both MMN and ERAN belong to a family of perisylvian negativites that mediate the processing of irregularities of auditory input. With this respect, the present results support the hypothesis of a strong adaptability and flexibility of fast and automatic cognitive processes in the human brain, probably indicating that the classical MMN framework might be expanded (at least with respect to the processing of major-minor tonal music) to the processing of complex, or syntactic, rules. Third, results support the hypothesis that processing of musical syntax as reflected in the ERAN is processed pre-attentively [5].

\section{REFERENCES}

1. Tervaniemi M, Medvedev SV, Alho K et al. Hum Br Mapp 10, 74-79 (2000).

2. Alain C, Achim A and Woods DL. Psychophysiology 36, 737-744 (1999)

3. Patel AD, Gibson E, Ratner J et al. J Cogn Neurosci 10, 717-733 (1998).

4. Koelsch S, Gunter T, Friederici AD et al. J Cogn Neurosci 12, 520-541 (2000).

5. Koelsch S, Schröger E and Gunter T. submitted (2001).

6. Koelsch S, Maess B and Fiederici AD. Neuroimage 11, 56 (2000).

7. Hahne A and Friederici AD. J Cogn Neurosci 11, 194-205 (1999).

8. Näätänen R. Attention and Brain Function. Hillsdale, NJ: Erlbaum, 1992.

9. Schröger E. Behav Res Methods Instr Comp 30, 131-145 (1998).

10. Krumhansl C and Kessler E. Psych Rev 89, 334-368 (1982).

11. Bharucha J and Krumhansl C. Cognition 13, 63-102 (1983).

12. Swain J. Musical Languages. UK: Norton, 1997.

13. Saarinen J, Paavilainen P, Schröger E et al. Neuroreport 3, 1149-1151 (1992).

14. Tervaniemi M, Maury S and Näätänen R. Neuroreport 5, 844-846 (1994).

15. Paavilainen P, Saarinen J, Tervaniemi M et al. Psychophysiology 9, 243-249 (1995).

16. Paavilainen P, Jaramillo $M$ and Näätänen R. Psychophysiology $\mathbf{3 5}$, 483-487 (1998).

17. Hindemith P. Unterweisung im Tonsatz, 1. Theoretischer Teil. Mainz: Schott; 1940.

18. Tiitinen H, May P, Reinkainen K and Näätänen R. Nature 372, 90-92 (1994).

19. Alho K. Ear Hear 16, 38-51 (1995).

20. Giard M, Perrin F and Pernier J. Psychophysiology 27, 627-640 (1990).

21. Alain C, Woods DL and Knight RT. Brain Res 812, 23-37 (1998).

22. Opitz B, Mecklinger A, von Cramon DY et al. Psychophysiology 36, 142-147 (1999).

23. Friederici AD, Wang Y, Herrmann C et al. Hum Br Map 11, 1-11 (2000).

Acknowledgement: The work was supported by the Leibniz Science Prize awarded to A.D. Friederici by the German Research Foundation. Full color maps, sound examples of the stimulation, and abstract with figures of the submitted article are available at www.stefan-koelsch.de 